\title{
Too low, too high. Is it the Roux-en-Y? Fluctuating thyroid function post obesity surgery
}

\author{
JD Crane (ST4 Diabetes and Endocrinology) and IN Scobie (Consultant Endocrinologist) Medway \\ Maritime Hospital, Windmill Road, Gillingham, Kent, ME7 5NY, UK
}

Morbid obesity (body mass index $>40 \mathrm{~kg} / \mathrm{m} 2$ ) is a growing global epidemic afflicting approximately $3 \%$ of the UK population. Hypothyroidism is a common hormone deficiency with a prevalence of approximately $2 \%$. With obesity surgery remaining the only intervention with the ability to bestow long term, significant reduction in excess body weight, there are increasing numbers of patients undergoing such procedures while on thyroid hormone replacement.

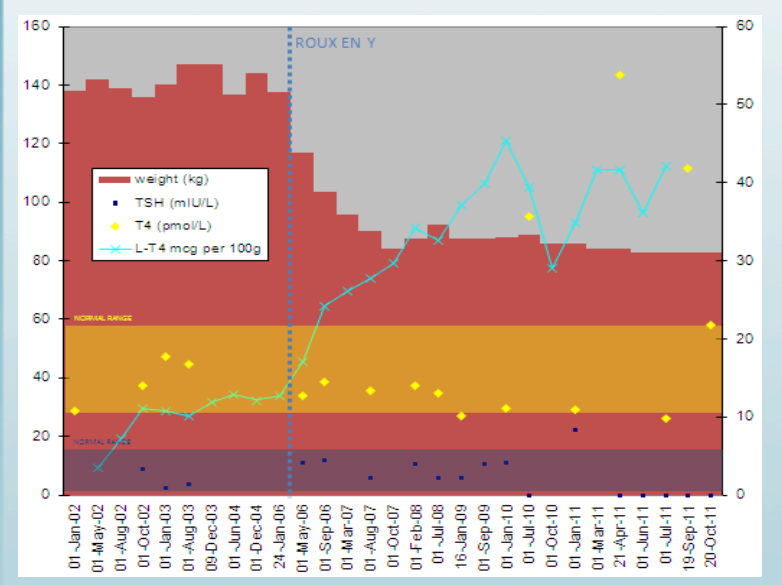

Our patient is a young woman in her 30's with autoimmune
hypothyroidism and coexistent super-morbid obesity with a peak BMI
of $54.9 \mathrm{~kg} / \mathrm{m} 2$. Euthyroidism was maintained with a levothyroxine dose
$175 \mathrm{mcg}$ daily ( $\mathrm{Imcg} / \mathrm{kg} /$ day). After failure of conservative measures,
she underwent weight loss surgery (proximal roux-en-y gastric
bypass). She subsequently achieved massive weight loss to reach a
new stable BMI of $32 \mathrm{~kg} / \mathrm{m} 2$. Following surgery, levothyroxine
requirements rapidly increased to fluctuate between 250 and $400 \mathrm{mcg}$
(2.9-4.5mcg/kg/day) with no single dose achieving a stable TSH. She
reported near-perfect adherence with appropriate separation of
levothyroxine and other medications including new nutritional
supplements. The presence of heterophile antibodies was assayed and
excluded.

Previous studies looking specifically at the bioavailability of levothyroxine following gastric bypass are limited. Reduced bioavailability was known to be a problem in older and more severely malabsorptive distal gastric bypass procedures [I,2], but reports of patients undergoing modern, proximal procedures have been mixed with at least one series demonstrating no difference [3]. On the other hand, gastric bypass is known to clearly impair bioavailability of certain other pharmaceuticals [4] with many different mechanisms being implicated:

Those drugs which undergo significant enterohepatic recirculation (like levothyroxine) would be likely to be most affected by changes in bile acid availability

The point of anastomosis and degree of shortening of the small bowel can impact the absorption of drugs whose uptake is maximal in the proximal small bowel or which have saturable uptake processes

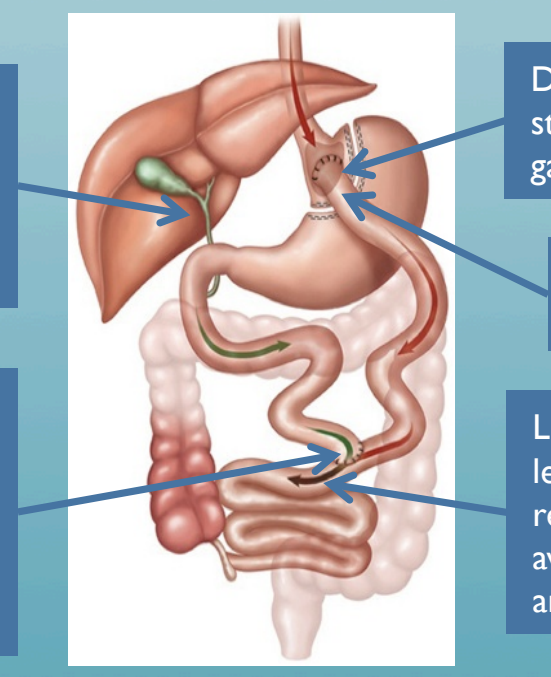

Disintegration of tablets in the stomach is affected by alteration of gastric motility

Dissolution of acidic drugs is impaired by increasing gastric $\mathrm{pH}$

Lipophilic molecules (like levothyroxine) are dispersed less readily due to reduction in bile acid availability in the linear and postanastomotic arms of the roux-en-y

The population of post-bariatric surgery patients in the endocrine clinic is growing. Issues with previously stable drug regimens are likely to become a more frequent feature of our practice. Awareness of and vigilance for such problems are key to preventing drug administration errors. When problems arise the mechanisms outlined above should be considered. Switching to liquid preparations or crushing tablets may mitigate inadequate disintegration. Dose increases, particularly of lipophilic drugs, may be necessary following bypass surgery. Following severely malabsorptive procedures, alternative administration routes (e.g. nasal, topical or rectal where available for individual drugs) should be considered.

References 1. Azizi FBelur R.Albano J. Malabsorption of thyroid hormone after jejunoileallbypass for 2. Bevan 15 , Munro IF

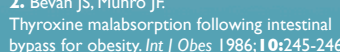
3. Rubio IGS, Galrao AL, Santo MA, Zanini AC, Medeiros-Neto G. Levothyroxine absorption in morbidly obese patients before and after roux-en-y gastric bypass (RYGG) surgery. Obes Surg 2012:22:253-258 4. Padwal R, Brocks D, Sharma AM. A systematic review of drug absorption
following bariatric surgery and its theoretict
implications. Obes Rev 2010;1 I:41-50 